\title{
Review of: "A structured ICA-based process for removing auditory evoked potentials"
}

\author{
Pedro C. Gordon ${ }^{1}$, Ulf Ziemann ${ }^{1}$ \\ 1 Eberhard-Karls-Universität Tübingen
}

Potential competing interests: The author(s) declared that no potential competing interests exist.

The recent paper by Ross et al. (2022) addresses an important issue pertaining to TMS-EEG studies, namely the proper control for undesired peripherally evoked potentials (PEPs) in the EEG response. PEPS constitute a considerable confounder in the analysis of EEG responses to the direct cortical activation by TMS, the TMS evoked potentials (TEPs) (Siebner et al. 2019; Belardinelli et al. 2019). This caveat to TMSEEG analysis has been described since the early developments of the technique, with PEPs caused by the considerable sensory input from TMS in the form of a high-intensity high-pitch "click" sound, and activation of skin nerve ending and muscles near the TMS target. PEPs manifest particularly prominently as a sequence of deflections around 100 and 200 ms after the TMS pulse, called N100-P200 complex (IImoniemi and Kicic 2010). Moreover, this issue has been recently brought into a new light, revealing the extent of the PEPs pervasiveness in TMS-EEG experiments, and the significant risks of misinterpreting them as the true TEPs (Conde et al. 2019).

Proposals like the present one by Ross et al. (2022) to remove PEPs from the recorded TMS-EEG response signal are therefore timely, and should be received with enthusiasm, but also with prudence and deliberation, in order to objectively determine their validity and applicability. At first, the method proposed by Ross et al. (2022) has the advantage that it does not involve a completely new tool that researchers would have to get familiar with. Instead, independent component analysis (ICA) is already part of the standard techniques to remove other artifacts from TMS-EEG data (Rogasch et al. 2017; Atluri et al. 2016). Moreover, the possibility of using ICA to also remove PEPs has already been considered previously, as mentioned by the authors, who propose to systematically establish and test the method with the described experiment.

In that experiment, in order to characterize the PEPs in the TMS-EEG signal, a sham procedure was applied to simulate the sensory inputs from the real TMS condition. A first observation is that the sensory input from the sham procedure most likely was not equivalent to the one from the real TMS condition, as it becomes clear from figure 1B, that displays a visibly lower response amplitude from the sham trials. The underestimation of the importance of somatosensory inputs in TME-EEG PEPs is already evident in the article's title, which only acknowledges the auditory components. This possibly influenced the choice of the electrical stimulation intensity parameters in the sham condition, which are too low to simulate the real TMS, in particular when considering that one of the TMS targets (the dorsolateral prefrontal cortex, DLPFC) 
is a sensitive scalp region. Indeed, many previous reports have encountered this same limitation (Conde et al. 2019; Gordon et al. 2018; Rocchi et al. 2021; Du et al. 2017), which points to the difficulty in devising a realistic sham control that accurately matches the sensory input from the real TMS. However, it is possible that the presented method by Ross et al. (2022) might not need such strict matching of sensory inputs: Regardless of differences in PEP amplitudes, the ICA may be able to identify the temporal and spatial characteristics of the PEPs, enabling the detection and removal of signal components containing the PEPS, even if the sensory input from the sham condition does not strictly match that from the real TMS. As a consequence, the authors show an apparently satisfying result in removing components of the TMSEEG response usually attributed to PEPs, resulting in the suppression of temporal and spatial signatures of the N100-P200 response, as demonstrated in figures 2, 4 and 6 of the article. The proper removal of this component seems to have been essential to reveal the presence of cortical responses likely evoked by the direct activation of the TMS, but which were obscured by the PEPs, such as a deflection around $120 \mathrm{~ms}$ located at the site of the stimulated left DLPFC, which is similar to our recent findings when targeting with TMS the primary motor cortex (Gordon et al. 2021).

Despite this apparent success in revealing potentially true TEPs from TMS activation, there is always the potential risk that the ICA procedure inevitably removes part of the TEP response in addition to the PEPS. One of the fundamental assumptions of ICA is the independence of the components, which cannot be postulated here due to the very nature of TEPS and PEPS, which are both time-locked to the TMS pulse and originate from cortical sources (Metsomaa, Sarvas, and IImoniemi 2014). Even though this has not been systematically analyzed, figure 1C in the article of Ross et al. (2022) displays some early deflections (starting at around $50 \mathrm{~ms}$ after the TMS pulse) that have been classified as PEPs. However, given their early latency, these components may well have been TEPs. Vice versa, it is also not completely possible from the present report to be certain that all components of PEPs have been removed, leading to the risk of misinterpreting components of PEPS as TEPS.

These limitations are even made more complicated by the necessary involvement of the investigator in visually determining what components are attributed to PEPs, which, as also acknowledged by the authors, entails a degree of subjectivity and inter-rater variability. This limitation is not present in other approaches, such as SSP-SIR (Biabani et al. 2019), which also uses responses to sham stimulation to inform the removal of PEPs from the TMS-EEG data; or the subtraction of sham responses evoked by thoroughly matched sensory inputs from the real TMS responses, which we proposed recently (Gordon et al. 2021). Unfortunately, all methods are subject to limitations, and presently there is no ground truth regarding TEP analysis and sham control. Nevertheless, a systematic comparison of the available methods should be helpful in shedding light into which techniques might be the best suited, hopefully leading to a more straightforward way of revealing the true EEG signatures of direct cortical activation by TMS.

\section{REFERENCES:}

- Atluri, S., M. Frehlich, Y. Mei, L. Garcia Dominguez, N. C. Rogasch, W. Wong, Z. J. Daskalakis, and F. 
Farzan. 2016. 'TMSEEG: A MATLAB-Based Graphical User Interface for Processing Electrophysiological Signals during Transcranial Magnetic Stimulation', Front Neural Circuits, 10: 78.

- Belardinelli, P., M. Biabani, D. M. Blumberger, M. Bortoletto, S. Casarotto, O. David, D. Desideri, A. Etkin, F. Ferrarelli, P. B. Fitzgerald, A. Fornito, P. C. Gordon, O. Gosseries, S. Harquel, P. Julkunen, C. J. Keller, V. K. Kimiskidis, P. Lioumis, C. Miniussi, M. Rosanova, S. Rossi, S. Sarasso, W. Wu, C. Zrenner, Z. J. Daskalakis, N. C. Rogasch, M. Massimini, U. Ziemann, and R. J. Ilmoniemi. 2019. 'Reproducibility in TMSEEG studies: A call for data sharing, standard procedures and effective experimental control', Brain Stimul, 12: 787-90.

- Biabani, M., A. Fornito, T. P. Mutanen, J. Morrow, and N. C. Rogasch. 2019. 'Characterizing and minimizing the contribution of sensory inputs to TMS-evoked potentials', Brain Stimul, 12: 1537-52.

- Conde, V., L. Tomasevic, I. Akopian, K. Stanek, G. B. Saturnino, A. Thielscher, T. O. Bergmann, and H. R. Siebner. 2019. 'The non-transcranial TMS-evoked potential is an inherent source of ambiguity in TMSEEG studies', Neuroimage, 185: 300-12.

- Du, X., F. S. Choa, A. Summerfelt, L. M. Rowland, J. Chiappelli, P. Kochunov, and L. E. Hong. 2017. 'N100 as a generic cortical electrophysiological marker based on decomposition of TMS-evoked potentials across five anatomic locations', Exp Brain Res, 235: 69-81.

- Gordon, P. C., D. Desideri, P. Belardinelli, C. Zrenner, and U. Ziemann. 2018. 'Comparison of cortical EEG responses to realistic sham versus real TMS of human motor cortex', Brain Stimul, 11: 1322-30.

- Gordon, P. C., D. B. Jovellar, Y. Song, C. Zrenner, P. Belardinelli, H. R. Siebner, and U. Ziemann. 2021. 'Recording brain responses to TMS of primary motor cortex by EEG - utility of an optimized sham procedure', Neuroimage, 245: 118708.

- Ilmoniemi, R. J., and D. Kicic. 2010. 'Methodology for combined TMS and EEG', Brain Topogr, 22: 233-48.

- Metsomaa, J., J. Sarvas, and R. J. Ilmoniemi. 2014. 'Multi-trial evoked EEG and independent component analysis', J Neurosci Methods, 228: 15-26.

- Rocchi, L., A. Di Santo, K. Brown, J. Ibanez, E. Casula, V. Rawji, V. Di Lazzaro, G. Koch, and J. Rothwell. 2021. 'Disentangling EEG responses to TMS due to cortical and peripheral activations', Brain Stimul, 14: 4-18.

- Rogasch, N. C., C. Sullivan, R. H. Thomson, N. S. Rose, N. W. Bailey, P. B. Fitzgerald, F. Farzan, and J. C. Hernandez-Pavon. 2017. 'Analysing concurrent transcranial magnetic stimulation and electroencephalographic data: A review and introduction to the open-source TESA software', Neuroimage, 147: 934-51.

- Ross, J. M., R. A. Ozdemir, S.J. Lian, P. J. Fried, E. M. Schmitt, S. K. Inouye, A. Pascual-Leone, and M. M. Shafi (2022) A structured ICA-based process for removing auditory evoked potentials. Sci Rep 12:1391.

- Siebner, H. R., V. Conde, L. Tomasevic, A. Thielscher, and T. O. Bergmann. 2019. 'Distilling the essence of TMS-evoked EEG potentials (TEPs): A call for securing mechanistic specificity and experimental rigor', Brain Stimul, 12: 1051-54. 
